\title{
Optimization of Fuzzy Species Pythagorean Transportation Problem under Preserved Uncertainties
}

\author{
Priyanka Nagar \\ Department of Mathematics, \\ Jaypee Institute of Information Technology, 201304, Noida, India. \\ E-mail: nagarpriyanka872@gmail.com \\ Pankaj Kumar Srivastava \\ Department of Mathematics, \\ Jaypee Institute of Information Technology, 201304, Noida, India. \\ Corresponding author: pankaj.srivastava@jiit.ac.in \\ Amit Srivastava \\ Department of Mathematics, \\ Jaypee Institute of Information Technology, 201304, Noida, India. \\ E-mail: amit.srivastava@jiit.ac.in
}

(Received on April 11, 2021; Accepted on October 12, 2021)

\begin{abstract}
The transportation of big species is essential to rescue or relocate them and it requires the optimized cost of transportation. The present study brings out an optimized way to handle a special class of transportation problem called the Pythagorean fuzzy species transportation problem. To deal effectively with uncertain parameters, a new method for finding the initial fuzzy basic feasible solution (IFBFS) has been developed and applied. To test the optimality of the solutions obtained, a new approach named the Pythagorean fuzzy modified distribution method is developed. After reviewing the literature, it has been observed that till now the work done on Pythagorean fuzzy transportation problems is solely based on defuzzification techniques and so the optimal solutions obtained are in crisp form only. However, the proposed study is focused to get the optimal solution in its fuzzy form only. Getting results in the fuzzy form will lead to avoid any kind of loss of information during the defuzzification process. A comparative study with other defuzzification-based methods has been done to validate the proposed approach and it confirms the utility of the proposed methodology.
\end{abstract}

Keywords- Fuzzy species transportation problems, Pythagorean fuzzy numbers, Initial fuzzy basic feasible solution, Pythagorean fuzzy modified distribution method.

\section{Introduction}

The transportation of big species is the deliberate movement of creatures by transport. Common groups of species which are transported comprise; zoological specimens; laboratory creatures; race farm animals; pets; and the other animals to rescue or relocate. Methods of transporting animals vary greatly from species to species. In last decade, the species movement in optimized time due to climate change has been thoughtfully studied by Madden et al. (2011). In continuation, Olden et al. (2011) tested whether managed relocation of freshwater species is ecologically sound or not and provided strategies for managed relocation. An interesting evaluation of species relocation programmers in Australia have been performed by Sheean et al. (2012). There are certain similar cases in real world which require an optimized species transportation for relocation. After reviewing the literature, it has been observed that the major part of the existing work contributes to species transportation with crisp values only. However, there are many cases having uncertain, inconsistent and imprecise data, which gives the inconsistency in results. Conditions of roads, 
fluctuating price of petrol and diesel and weather conditions are some examples, which increases the impreciseness of the product. To describe this uncertainty or fuzziness, fuzzy numbers have been introduced in transportation problems. In the present study, Pythagorean fuzzy numbers (PFNs) based species transportation problems have been considered due to large domain size of these numbers as similar to the intuitionistic fuzzy sets they define both the membership and nonmembership values for a component, but additionally, the square sum of the membership and nonmembership degrees is maximum one.

To overcome the situation of inconsistency, one can convert the crisp data in fuzzy numbers using the theory of fuzzy sets proposed by Zadeh (1965). In this paper, author defined the concept of fuzzy sets as well as union, intersection and complement etc. are extended to fuzzy sets. The basics of fuzzy sets, fuzzy membership and defuzzification have been described by Bisht et al. (2018). Also, this chapter provides different feature of fuzzy manufacturing system and some standard fuzzy logic applications. Fuzzy logic, fuzzy sets and application of fuzzy computing are discussed by Srivastava and Bisht (2019). This chapter describes that fuzzy logic can handle vagueness, imprecision and uncertainty. Two authors, Bellman and Zadeh (1970), introduced the theory of fuzzy sets in multicriteria decision-making problems. The duo suggested that the decision maker (DM) gives the degree to which an alternative satisfies any criterion. However, in practical multicriteria decision-making process, the DM provides not only the degree by which an alternative satisfies the criterion but also gives the degree of dissatisfaction to the alternative. To the solution of this, Atanassov (1986) extend the concept of fuzzy set to develop intuitionistic fuzzy set (IFS) and this IFS is characterized by membership and non-membership degree and this fuzzy set also satisfies one important condition which is the sum of membership and non-membership degree is equal or less than one i.e., $\mu+v \leq 1$. Later, it has been found that there are many real-life decision problems which cannot be solved using IFS theory as in those problems, the addition of support degree and against degree is equal to or greater than one i.e., $\mu+v \geq 1$. Then, Yager (2013) introduced Pythagorean fuzzy set (PFS) which is a generalization of IFS, and this set satisfies the condition which is the sum of squares of support degree and against degree is equal to or less than one i.e., $\mu^{2}+v^{2} \leq 1$. Obviously, PFS cover more area than IFS, the ability to handle the uncertainty by PFS is more powerful than IFS. That is why, the PFNs are taken in this article to optimize our problem.

Transportation models are very useful in real world problems. Hitchcock (1941) is the first person who introduced the transportation model to the world. In this model, the cost of supply, demand and transportation of any consignment has taken in precise form. However, in practical situations, it has been examined that it is impossible to have these quantities in crisp form. Various transportation problems have been solved using fuzzy programming Zimmermann (1978). In last few decades, fuzzy optimization has gained attention among researchers as it has various applications in transportation problems, traffic assignment problem and travelling salesman problem. Number of attempts have been done by many researchers to evaluate transportation problems in fuzzy environment. Fuzzy transportation problems (FTPs) have been developed by Chanas et al. (1984). Fully interval integer transportation problems (Akilbasha et al., 2018) are solved using an exact method. In this problem, author developed two different transportation problems from a single fuzzy transportation problem by using mid-width method and obtained optimal transportation cost in triangular fuzzy numbers. Bharati and Singh (2018) used intervalvalued intuitionistic fuzzy sets (IVIF) instead of fuzzy and intuitionistic fuzzy sets and shows that the cost of transportation using IVIF sets is lesser than fuzzy and intuitionistic fuzzy sets. They also 
define interval-valued membership and non-membership of imprecise parameters. Liu et al. (2014) proposed three types of defuzzification techniques for type-2 fuzzy variables to solve solid transportation problem and tabu search algorithm is used to find approximate solution. Type-2 fuzzy variables are used by Kundu et al. (2014) to solve two fixed charge transportation problems. To defuzzify type-2 fuzzy values into type-1 fuzzy values, critical value-based reduction method is used and to complete the defuzzification, centroid method is also applied. Li and Lai (2000) proposed fuzzy compromise programming approach to evaluate multi-objective transportation problems and using the proposed approach one can get a non-dominated compromise and optimal solution. Singh and Yadav (2016), defined accuracy function for membership and non-membership functions of triangular intuitionistic fuzzy numbers to solve type-2 fuzzy intuitionistic transportation problems. Basic feasible solution is found out using the proposed ordering of triangular intuitionistic fuzzy numbers. Authors concluded that the proposed methods can be extended for solving those transportation problems in which data is non-symmetrical form. Arora (2018), proposed an approach to solve interval-valued fuzzy fractional transportation problem. Author proved that interval-valued fuzzy fractional transportation problem can be converted into linear fuzzy transportation problem. Also, author used simplex method to find the optimum solution. Hashmi et al. (2019), evaluated two stage fixed charge transportation problem with multiple objectives. In this paper, authors adopted fuzzy goal programming technique with linear and non-linear membership functions to solve multi-objective problem and significant results are found using non-linear membership functions. Some other methods are also present in the literature by which fuzzy transportation problems have been solved likewise extension principle-based technique has adopted to calculate the cost of the problem having fuzzy data Liu and Kao (2004). In this article, authors discussed two types of transportation problem in which one problem is having inequality constraints and the other is having equality constraints. Ranking function (Kaur and Kumar, 2011) has been applied to optimize the problem. In this study, authors proposed a new technique which is based on classical method to find initial fuzzy basic feasible solution and used generalized trapezoidal fuzzy numbers as parametric values. A non-fuzzy method (Pandian and Natarajan, 2010) has been used to optimize the transportation problem having all entries in interval form. Also, a separation method is developed which is based on zero-point method. This method provides an optimal solution for fully interval transportation problem. Transportation problem having all the parameters in fuzzy form, determined using zero point and zero suffix method by Ngastiti et al. (2018). In this study, robust ranking technique is used for defuzzification and it has been shown that zero suffix method takes less iteration than zero-point method, whereas allocation and optimal cost are same. A method known as Modified best candidate has been proposed to find solution for fuzzy transportation problem Dinagar and Keerthivasan (2018). This method can be used for any type of matrices to solve fuzzy transportation problem. Also, author concluded that this technique reduces the overall calculation time and hence obtain the optimal solution very quickly. The concept of optimal solution of TPs in which transportation cost is in fuzzy value has been proposed by Chanas and Kuchta (1996) and to obtain the optimal solution, authors also developed an algorithm. The technique provides a set of elementary solutions which is very useful for decision maker. Interval databased transportation problems (IBTPs) have been solved using a defuzzification scheme by Bisht and Srivastava (2018). In this paper, authors proposed a new trisectional approach to convert IBTP into FTP and a unique defuzzification method based on incenter of triangle is developed to convert triangular fuzzy numbers into crisp numbers. New approaches are suggested to optimize fuzzy transportation cost by Bisht and Srivastava (2017). A unique conversion approach is proposed to transform triangular fuzzy numbers into trapezoidal fuzzy numbers and a ranking scheme is also developed to convert trapezoidal fuzzy numbers into crisp numbers. In this study, author concluded that the present algorithms are better and fast than 
classical methods. Two new techniques are proposed with new representation of trapezoidal fuzzy numbers called JMD representation of trapezoidal fuzzy numbers by Kumar and Kaur (2011) to find an optimal solution. In this study, it has been shown that if the new representation of trapezoidal fuzzy numbers is taken instead of existing representation and the proposed method is applied then the optimal transportation cost is same but the total number of constraints is less. New approaches based on classical methods are proposed for finding the initial basic feasible solution by Kaur and Kumar (2012). In this paper, cost of transportation is taken in generalized trapezoidal fuzzy numbers and supply and demand are in crisp form. Generalized fuzzy numbers are used to solve a special type of transportation problem instead of normal fuzzy numbers by Samuel and Venkatachalapathy (2013). The study shows that the proposed method is far better than GFNWCM, GFLCM and GFVAM to solve FTP and proposed method requires simple calculations which are easy to understand. Generalized form of trapezoidal fuzzy numbers has been taken to find the solution of FTPs by Ebrahimnejad (2014). In this study, author solved the same problem taken by Kaur and Kumar (2012) and shown that the same optimal solution can be obtained without solving any FTP. In this way, author reduces the computational complexity of the existing method. Ebrahimnejad (2015) proposed a two-step method to obtain fuzzy optimal solution using nonnegative triangular numbers. In first step, FTP is converted into a linear programming problem with fuzzy costs and crisp constraints and in second step a new decomposition technique is used to convert the resulting problem into three transportation problems. Gani and Razak (2006) optimized transportation problems having fuzzy values in two stages and to find a fuzzy solution, parametric approach is used. The optimal transportation cost is found by minimizing the sum of transportation cost obtained in two stages. Generalized fuzzy numbers are discussed by several authors (Bisht and Srivastava, 2019a; Bisht and Srivastava, 2019b; Chhibber et al., 2019; Mathur and Srivastava 2019; Nagar et al., 2019).

The transportation of species from one habitat to another is the careful movement of creatures through optimized transportation. To the best of author's knowledge, no optimization model is present in existing literature of Pythagorean fuzzy species transportation problems in which the optimum solution has obtained in fuzzy values. This research gap has motivated the authors to come up with a new technique, which can optimize the transportation problem in Pythagorean fuzzy environment.

Main contributions of the present work are as follows:

- In the present work, two new unique schemes have been proposed for finding the optimal solution of the Pythagorean fuzzy transportation problems.

- In the proposed methods, PFNs have taken and to obtain the IFBFS of Pythagorean fuzzy species transportation problem (PFSTP), a new technique is developed.

- To test the optimality of IFBFS, a new method named as Pythagorean fuzzy Modified distribution (PFMODI) method is developed. The proposed techniques are easy to understand and easy to use by the decision-makers.

The current work is organized as: Some basic definitions are discussed in section 2. Types of fuzzy sets and Pythagorean fuzzy numbers are described in section 3. Section 4 provides some properties of PFNs. New property of PFNs and comparison of two PFN are given in section 5. In section 6, transportation problem in fuzzy environment has been described and after that, two new ideas have been proposed to optimize Pythagorean fuzzy transportation problems in section 7 and section 8 , then methodology of the work has been presented in section 9. Validity of the current work has 
been checked through two problems and section 10 provides the comparative study. Last section provides the conclusion of the manuscript.

\section{Basic Definitions}

This section provides some fundamental definitions:

\subsection{Definition}

Take a set $\eta$ such that $\mu_{\eta}(y): \eta \rightarrow[0,1]$. The set $\eta^{\dagger}$ is defined as fuzzy set, if the membership function $\mu_{\eta}(y)$ such that $\eta^{\dagger}(y)=\left\{\left(y, \mu_{\eta}(y): y \in \eta\right.\right.$ and $\left.\left.\mu_{\eta}(y) \in[0,1]\right)\right\}$.

\subsection{Definition}

A fuzzy set is defined as normal fuzzy set if its maximal degree of membership is 1 i.e, $\mu_{\eta^{\dagger}}(y)=1$

\subsection{Definition}

A fuzzy set is said to be convex fuzzy set iff it satisfies the following condition $\mu_{\eta}\left(\delta y_{1}+(1-\delta) y_{2}\right) \geq \min \left(\mu_{\eta}\left(y_{1}\right), \mu_{\eta}\left(y_{2}\right)\right)$.

\subsection{Definition}

A fuzzy set having the properties of normal as well as convex fuzzy set is defined as fuzzy number. Moreover, the membership function has piecewise continuity.

\subsection{Definition}

Any fuzzy number $T=\left(l_{1}, l_{2}, l_{3}, l_{4}\right)$ is said to be a Trapezoidal fuzzy number if the membership function is defined as follows:

$$
\mu_{T}(y)=\left\{\begin{array}{cc}
\frac{y-l_{1}}{l_{2}-l_{1}}, & l_{1} \leq y \leq l_{2}, \\
1, & l_{2} \leq y \leq l_{3}, \\
\frac{y-l_{4}}{l_{3}-l_{4}}, & l_{3} \leq y \leq l_{4}, \\
0, \text { otherwise. }
\end{array}\right.
$$

\section{Types of Fuzzy Sets and Pythagorean Fuzzy Numbers}

\subsection{Intuitionistic Fuzzy Sets}

Atanassov (1986) proposed the concept of IFS to reduce the dubiousness. IFS dealt with the membership degree and non-membership degree along with the hesitation degree of the element.

Let $\mathrm{S}$ be a non-empty set and an IFS $\chi$ in $\mathrm{S}$ is defined as,

$\chi=\left\{\left\langle y, \mu_{\chi}(y), v_{\chi}(y)\right\rangle \mid y \in S\right\}$

where, 
$\mu_{\chi}(y): \mathrm{S} \rightarrow[0,1]$ represents the belongingness degree and $v_{\chi}(y): \mathrm{S} \rightarrow[0,1]$ represents the non- belongingness degree of $y \in S$ to the set $\chi$, respectively, for every $y \in S$ it satisfies the relation,

$0 \leq \mu_{\chi}(y)+v_{\chi}(y) \leq 1$

Following relation gives the degree of non-determinacy

$h_{\chi}(y)=1-\left(\mu_{\chi}(y)+v_{\chi}(y)\right)$

\subsection{Pythagorean Fuzzy Sets}

In some cases, it can be seen that on adding membership degree and non-membership degree the resultant is bigger than one. To handle this type of condition, (Yager and Abbasov, 2013; Yager, 2013; Yager, 2014) recently discovered Pythagorean fuzzy set, which defines that on adding square of membership degree and square of non-membership degree, the resultant is less than or equal to 1.

Suppose $\Psi$ be a universal set and a Pythagorean fuzzy set $\tau$ in $\Psi$ is defined as

$\tau=\left\{\left\langle y, \mu_{\tau}(y), v_{\tau}(y)\right\rangle \mid y \in \Psi\right\}$

where, $\mu_{\tau}(y): \Psi \rightarrow[0,1]$ represents the degree of membership and $v_{\tau}(y): \Psi \rightarrow[0,1]$ represents the degree of non-membership of $y \in \Psi$ to the set $\tau$, respectively, for every $y \in \Psi$ it satisfies the condition

$\left(\mu_{\tau}(y)\right)^{2}+\left(v_{\tau}(y)\right)^{2} \leq 1$

Using the following equation, the degree of hesitation is evaluated

$\pi_{\tau}(y)=\sqrt{1-\left(\mu_{\tau}(y)\right)^{2}-\left(v_{\tau}(y)\right)^{2}}$

\subsection{Pythagorean Fuzzy Numbers}

A PFN $\alpha=\left(y, \mu_{\tau}(y), v_{\tau}(y)\right)$ defined on $\boldsymbol{R}$ (set of real numbers). Mathematically, its membership and non-membership functions are defined as

$$
\mu_{\tau}(y)=\left\{\begin{array}{l}
\mathrm{O}, \text { for } y<b_{1} \\
f_{\tau}(y), \text { for } b_{1} \leq y \leq b_{2}, \\
1, \text { for } y=b_{2} \\
g_{\tau}(y), \text { for } b_{2} \leq y \leq b_{3} \\
0, \text { for } y>b_{3}
\end{array}\right.
$$


$\nu_{\tau}(y)=\left\{\begin{array}{l}1, \text { for } y<b_{1}^{\prime}, \\ \phi_{\tau}(y), \text { for } b_{1}^{\prime} \leq y \leq b_{2}, 0 \leq\left(f_{\tau}(y)\right)^{2}+\left(\phi_{\tau}(y)\right)^{2} \leq 1 \\ 0, \text { for } y=b_{2}, \\ \psi_{\tau}(y), \text { for } b_{2} \leq y \leq b_{3}^{\prime}, 0 \leq\left(g_{\tau}(y)\right)^{2}+\left(\psi_{\tau}(y)\right)^{2} \leq 1 \\ 1, \text { for } y>b_{3}^{\prime}\end{array}\right.$

where, $\left(\mu_{\tau}(y)\right)^{2}+\left(v_{\tau}(y)\right)^{2} \leq 1$ and $b_{1}, b_{2}, b_{3}, b_{1}^{\prime}, b_{3}^{\prime} \in \mathbb{R}$ such that $b_{1}^{\prime} \leq b_{1} \leq b_{2} \leq b_{3} \leq b_{3}^{\prime}$ and $f_{\tau}(y), g_{\tau}(y), \phi_{\tau}(y), \psi_{\tau}(y)$ are functions from $\mathbb{R} \rightarrow[0,1], f_{\tau}(y)$ and $\phi_{\tau}(y)$ are strictly increasing functions and $g_{\tau}(y)$ and $\psi_{\tau}(y)$ are strictly decreasing functions.

\section{Properties of Pythagorean Fuzzy Numbers}

Let $\alpha=\left(\mu_{1}, v_{1}\right)$ and $\beta=\left(\mu_{2}, v_{2}\right)$ are two PFNs. Then the arithmetic operations on PFNs are as follows

(i) $\alpha \oplus \beta=\left(\sqrt{\left(\mu_{1}^{2}+\mu_{2}^{2}-\mu_{1} \cdot \mu_{2}\right)}, v_{1} \cdot v_{2}\right)$;

(ii) $\alpha \otimes \beta=\left(\mu_{1} \cdot \mu_{2}, \sqrt{\left(v_{1}^{2}+v_{2}^{2}-v_{1} \cdot v_{2}\right)}\right)$;

(iii) $\alpha \Theta \beta=\left(\sqrt{\frac{\mu_{1}^{2}-\mu_{2}^{2}}{1-\mu_{2}^{2}}}, \frac{v_{1}}{v_{2}}\right)$ if $\mu_{1} \geq \mu_{2}, v_{1} \leq \min \left(v_{2}, \frac{v_{2} \pi_{1}}{\pi_{2}}\right)$;

(iv) $\alpha \Theta \beta=\left(\frac{\mu_{1}}{\mu_{2}}, \sqrt{\frac{v_{1}^{2}-v_{2}^{2}}{1-v_{2}^{2}}}\right)$ if $\mu_{1} \leq \min \left(\mu_{2}, \frac{\mu_{2} \pi_{1}}{\pi_{2}}\right), v_{1} \geq v_{2}$;

(v) $\kappa . \alpha=\left(\sqrt{1-\left(1-\mu_{1}^{2}\right)^{\kappa}}, v^{\kappa}\right)$.

where, $\kappa$ is a scalar quantity.

\section{Proposed Property and Comparison of Pythagorean Fuzzy Numbers}

\subsection{Proposed Additive Identity of Pythagorean Fuzzy Numbers}

Let $\left(\mu_{1}, v_{1}\right)$ and $\left(\mu_{2}, v_{2}\right)$ be any two PFN. The PFN $\left(\mu_{2}, v_{2}\right)$ is called an additive identity of PFN if, $\left(\mu_{1}, v_{1}\right) \oplus\left(\mu_{2}, v_{2}\right)=\left(\mu_{1}, v_{1}\right)=\left(\mu_{2}, v_{2}\right) \oplus\left(\mu_{1}, v_{1}\right)$, then $\left(\mu_{2}, v_{2}\right)=(0,1)$.

where, $(0,1)$ represents the proposed additive identity for PFNs.

\subsection{Ordering of Pythagorean Fuzzy Numbers}

Decision maker (DM) have to compare fuzzy numbers, in order to give correct decision. Therefore, in decision-based situations, comparison is utmost essential. 
Let $\alpha=\left(\mu_{1}, v_{1}\right)$ and $\beta=\left(\mu_{2}, v_{2}\right)$ are two PFNs. Zhang (2016) developed ordering of two PFNs as $\alpha \geq \beta$ iff $\mu_{1} \geq \mu_{2}$ and $v_{1} \leq v_{2}$, where $\geq$ bigger than or indifferent to.

\section{Species Relocation Transportation Problems in Fuzzy Environment}

Till date it is understood that species relocation transportation problem has influencing role in real life. In conventional transportation problem, all the parameters such as demand, supply and transportation cost are taken in precise form. However, there are many situations where one can see the impreciseness in these parameters. For example, climatic conditions, roads condition, traffic and prices of petrol and diesel play the most important role to increase the impreciseness of the consignment and these fuzzy species transportation problems in which the parameters assume imprecise values cannot be solved using conventional transportation model. Imprecise values cannot be solved using conventional transportation model (Dinagar and Palanivel, 2009).

To overcome this impreciseness, Bellman and Zadeh (1970), implement the theory of fuzzy set to transportation problem and this has been used in the notion of Fuzzy Transportation Problems (FTPs) or one can say transportation problem in fuzzy environment and this approach has been adopted here in this study of Pythagorean fuzzy species transportation problem (PFSTP).

Mathematical formulation of PFSTP is given below:

$$
\operatorname{Min} \delta=\sum_{i=1}^{m} \sum_{j=1}^{m} \tilde{p}_{i j} z_{i j}
$$

subject to,

$$
\begin{aligned}
& \sum_{\substack{j=1 \\
m}}^{m} z_{i j}=\tilde{r}_{i}, i=1,2, \ldots, m \\
& \sum_{i=1}^{m} z_{i j}=\tilde{s}_{j}, j=1,2, \ldots, m \\
& z_{i j} \geq 0, i=1,2, \ldots, m, j=1,2, \ldots, m
\end{aligned}
$$

Fuzzy transportation problems have solved by several ranking techniques (Mathur et al., 2016; Mathur et al., 2018; Srivastava and Bisht, 2018). The present study is focused on optimized transportation of big species in fuzzy environment.

\section{Proposed Method to find the IFBFS of PFSTP}

If the given problem is not balanced, then add fuzzy dummy row or fuzzy dummy column to make it balanced one.

STEP 1. Using the ordering property of two PFNs, choose the smallest element in each row and then minus this smallest element from each cell of the respective rows.

STEP 2. For each column of the obtained matrix, again choose the minimum element in each column of the obtained matrix and then minus this smallest element from each cell of the respective 
columns. After doing STEP 1. and STEP 2., reduced Pythagorean fuzzy transportation problem is attained in which $(0,1)$ is additive identity.

STEP 3. Now add the fuzzy cost of all rows and all columns of the resulting matrix to obtain penalties.

STEP 4. After selecting the highest penalty of the resulting matrix, allocate that cell in the original matrix in which the fuzzy cost is minimum.

STEP 5. Repeat step 3 and step 4 until all demands and supplies have adjusted.

\section{Proposed Method for Finding Optimal Solution}

To attain the appropriate solution of IFBFS, authors proposed a unique technique.

STEP 1. Find the values of the variables $\omega_{i}$ and $\zeta_{j}$, using the relation $\omega_{i}+\zeta_{j}=\Omega_{i j}$.

STEP 2. Use $\Delta_{i j}=\omega_{i}+\zeta_{j}-\Omega_{i j}$, to find out the opportunity cost.

STEP 3. Criteria for checking the opportunity cost

- If the sum of the opportunity cost of the unoccupied cell is greater than or equal to 1 , the obtained solution is the appropriate solution.

- If the total opportunity cost of an inactive cell is less than 1 , the solution provided is not an appropriate solution and further investigation into travel costs is possible.

STEP 4. Choose that inactive cell in which the total opportunity cost is less than 1 as well as the most positive and include that inactive cell in the next step.

STEP 5. Make a closed loop for the unused cell chosen in step 4. Only right-angle turn is allow making this path at used cells and at the unused cell.

STEP 6. Marked sign of plus and sign of minus alternatively at the inactive cells on the corner place on the loop. Start with assigning sign of plus at the inactive cell.

STEP 7. The very small PFN with a negative place on the passage shows the units of the product, which are going to, sent to the entry point.

STEP 8. Now, add this small PFN to those cells of the loop having plus signs, and remove this small number from those cells having minus sign. By doing this, an inactive cell turns to an active cell.

STEP 9. The whole method repeated, until an appropriate result found.

\section{Methodology}

The whole procedure of the paper explained in this section. The steps involved in finding the optimal solution of the given problem are as follows:

STEP 1. Transform the problem into tabular form. 
STEP 2. Initial fuzzy basic feasible solutions found out using proposed method and the steps are below:

- Find the smallest PFN in each row and then subtract this smallest PFN from each entry of the respective rows.

- Now, find the smallest PFN in each column of the matrix, obtained after STEP A and subtract this smallest number from each entry of the respective columns.

- Sum of fuzzy costs of each row and each column of the obtained matrix gives the penalty. Choose the highest penalty of the obtained matrix and use this penalty to allocate the cell of the original matrix.

- Repeat the technique, until the original matrix reduces to the order $1 \times 1$.

STEP 3. To test the optimality of obtained IFBFS, a new method has proposed which involves the following steps:

- Dual variables $\omega_{i}$ and $\zeta_{j}$ are found out by the equation $\omega_{i}+\zeta_{j}=\Omega_{i j}$.

- Now, find the opportunity cost for unoccupied cells using the relation $\Delta_{i j}=\omega_{i}+\zeta_{j}-\Omega_{i j}$.

- That unoccupied cell whose sum of opportunity cost is less than 1 and also have most positive value has been selected to include in upcoming step.

- Draw a closed path from the selected unoccupied cell and assign addition and subtraction sign alternatively.

- Next, choose the least PFN among all the PFNs, which are at negative positions in the path, then add this number to those allocations where a plus sign assigned, and subtract from those allocations where a minus sign assigned. In this way, an unoccupied cell converts into occupied cell.

- Repeat the technique, until the optimal solution attained.

\section{Illustrations}

This section provides two numerical problems based on optimization of species relocation transportation cost to show the effectiveness of the proposed techniques.

Illustration 1. Species Pythagorean fuzzy transportation problem of type-1 represented in Table 1. Here $R_{1}, R_{2}$ and $R_{3}$ represents breeding domain and $S_{1}, S_{2}, S_{3}$ and $S_{4}$ are non-breeding domain in any ecosystem. Breeding domain have 26, 24 and 30 availabilities respectively and non-breeding domain have 17, 23, 28 and 12 requirements respectively. The cost of transporting species from one domain to another domain is given in Table 1 in PFN.

Table 1. Availability and requirement detail.

\begin{tabular}{|c|c|c|c|c|c|}
\hline & $\mathbf{S}_{\mathbf{1}}$ & $\mathbf{S}_{\mathbf{2}}$ & $\mathbf{S}_{\mathbf{3}}$ & $\mathbf{S}_{\mathbf{4}}$ & Availability \\
\hline $\mathbf{R}_{\mathbf{1}}$ & $(0.4,0.7)$ & $(0.5,0.6)$ & $(0.8,0.3)$ & $(0.6,0.4)$ & 26 \\
\hline $\mathbf{R}_{\mathbf{2}}$ & $(0.7,0.3)$ & $(0.4,0.6)$ & $(0.6,0.5)$ & $(0.7,0.3)$ & 24 \\
\hline $\mathbf{R}_{\mathbf{3}}$ & $(0.6,0.4)$ & $(0.8,0.4)$ & $(0.2,0.9)$ & $(0.9,0.1)$ & 30 \\
\hline Requirement & 17 & 23 & 28 & 12 & \\
\hline
\end{tabular}

Solution: The obtained IFBFS for illustration 1, after applying the proposed algorithm is Attained $b_{11}=15, b_{14}=11, b_{22}=23, b_{24}=1, b_{31}=2, b_{33}=28$ IFBFS has shown in Table 2 . 
Table 2. Attained IFBFS.

\begin{tabular}{|c|c|c|c|c|c|}
\hline & $\mathbf{S}_{\mathbf{1}}$ & $\mathbf{S}_{\mathbf{2}}$ & $\mathbf{S}_{\mathbf{3}}$ & $\mathbf{S}_{\mathbf{4}}$ & Availability \\
\hline $\mathbf{R}_{\mathbf{1}}$ & $\mathbf{1 5}(0.4,0.7)$ & $(0.5,0.6)$ & $(0.8,0.3)$ & $\mathbf{1 1}(0.6,0.4)$ & 26 \\
\hline $\mathbf{R}_{\mathbf{2}}$ & $(0.7,0.3)$ & $\mathbf{2 3}(0.4,0.6)$ & $(0.6,0.5)$ & $\mathbf{1}(0.7,0.3)$ & 24 \\
\hline $\mathbf{R}_{\mathbf{3}}$ & $\mathbf{2}(0.6,0.4)$ & $(0.8,0.4)$ & $\mathbf{2 8}(0.2,0.9)$ & $(0.9,0.1)$ & 30 \\
\hline Requirement & 17 & 23 & 28 & 12 & \\
\hline
\end{tabular}

The initial fuzzy transportation cost $=(0.99988,0)$ units.

Now to attain the optimal result of given Pythagorean FTP, proposed Pythagorean fuzzy Modi method has been applied in the IFBFS and shown in Table 3 and Table 4.

Taking $\omega_{1}=(0,1)$, the values of rest of the variables i.e., $\omega_{i}{ }^{\prime} s$ and $\zeta_{j}{ }^{\prime} s$ are find out. Here $(0,1)$ is additive identity.

Table 3. First table for finding opportunity cost.

\begin{tabular}{|c|c|c|c|c|}
\hline & $\zeta_{1}=(0.4,0.7)$ & $\zeta_{2}=(0.232,0.8)$ & $\zeta_{3}=(0.419,0.847)$ & $\zeta_{4}=(0.6,0.4)$ \\
\hline$\omega_{1}=(0,1)$ & $\mathbf{1 5}(0.4,0.7)$ & $(0.5,0.6)$ & $(0.8,0.3)$ & $\mathbf{1 1}(0.6,0.4)$ \\
\hline$\omega_{2}=(0.45,0.75)$ & $(0.7,0.3)$ & $\mathbf{2 3}(0.4,0.6)$ & $(0.6,0.5)$ & $\mathbf{1}(0.7,0.3)$ \\
\hline$\omega_{3}=(0.487,0.571)$ & $\mathbf{2}(0.6,0.4)$ & $(0.8,0.4)$ & $\mathbf{2 8}(0.2,0.9)$ & $(0.9,0.1)$ \\
\hline
\end{tabular}

Table 4. Second table for finding opportunity cost.

\begin{tabular}{|c|c|c|c|c|}
\hline & $\zeta_{1}=(0.4,0.7)$ & $\zeta_{2}=(0.232,0.8)$ & $\zeta_{3}=(0.254,0.864)$ & $\zeta_{4}=(0.6,0.4)$ \\
\hline$\omega_{1}=(0,1)$ & $\mathbf{1 7}(0.4,0.7)$ & $(0.5,0.6)$ & $(0.8,0.3)$ & $\mathbf{9}(0.6,0.4)$ \\
\hline$\omega_{2}=(0.45,0.75)$ & $(0.7,0.3)$ & $\mathbf{2 1}(0.4,0.6)$ & $(0.6,0.5)$ & $\mathbf{3}(0.7,0.3)$ \\
\hline$\omega_{3}=(0.787,0.5)$ & $\mathbf{2}(0.6,0.4)$ & $(0.8,0.4)$ & $\mathbf{2 8}(0.2,0.9)$ & $(0.9,0.1)$ \\
\hline
\end{tabular}

Using proposed method for finding optimal solution, the optimal basic variables and optimal solution is

$$
b_{11}=17, b_{14}=9, b_{22}=21, b_{24}=3, b_{32}=2, b_{33}=28
$$

Hence, the optimal solution using proposed method $=(0.99999,0)$ units.

It is clear from the above calculation that if the species in $R_{1}$ transported to $S_{1}$ and $S_{4}$, species in $R_{2}$ transported to $S_{2}$ and $S_{4}$ and species in $R_{3}$ transported to $S_{1}$ and $S_{3}$; the obtained cost of transportation is optimal.

The optimal transportation cost found out in fuzzy number, showing that there is no loss of any information. 
Illustration 2. Species Pythagorean fuzzy transportation problem of type-2 represented in Table 5. Here $T_{1}, T_{2}$ and $T_{3}$ represents breeding domain and $J_{1}, J_{2}, J_{3}$ and $J_{4}$ are non-breeding domain in any ecosystem. Breeding domain have $(0.7,0.4),(0.8,0.2)$ and $(0.9,0.1)$ availability respectively and non-breeding domain have $(0.4,0.7),(0.7,0.3),(0.6,0.5)$ and $(0.3,0.8)$ requirement respectively. The cost of transporting species from one domain to another domain is given in Table 5 .

Table 5. Availability and requirement detail.

\begin{tabular}{|c|c|c|c|c|c|}
\hline & $\mathbf{J}_{\mathbf{1}}$ & $\mathbf{J}_{\mathbf{2}}$ & $\mathbf{J}_{\mathbf{3}}$ & $\mathbf{J}_{\mathbf{4}}$ & Availability \\
\hline $\mathbf{T}_{\mathbf{1}}$ & 0.0355 & 0.0545 & 0.0775 & 0.0635 & $(0.7,0.4)$ \\
\hline $\mathbf{T}_{\mathbf{2}}$ & 0.056 & 0.07 & 0.026 & 0.07 & $(0.8,0.2)$ \\
\hline $\mathbf{T}_{\mathbf{3}}$ & 0.074 & 0.0815 & 0.06 & 0.09 & $(0.9,0.1)$ \\
\hline Requirement & $(0.4,0.7)$ & $(0.7,0.3)$ & $(0.6,0.5)$ & $(0.3,0.8)$ & \\
\hline
\end{tabular}

Solution: The obtained IFBFS for illustration 2, after applying the proposed algorithm is,

$$
\begin{aligned}
& b_{14}=(0.7,0.4), b_{21}=(0.4,0.7), b_{22}=(0.57475,0.57142), \\
& b_{23}=(0.6,0.5), b_{32}=(0.48828,0.525), b_{33}=(0.86632,0.19047)
\end{aligned}
$$

Attained IFBFS has shown in Table 6.

Table 6. Attained IFBFS.

\begin{tabular}{|c|c|c|c|c|c|}
\hline & $\mathbf{J}_{\mathbf{1}}$ & $\mathbf{J}_{\mathbf{2}}$ & $\mathbf{J}_{\mathbf{3}}$ & $\mathbf{J}_{\mathbf{4}}$ & Availability \\
\hline $\mathbf{T}_{\mathbf{1}}$ & 0.0355 & 0.0545 & 0.0775 & $(\mathbf{0 . 7 , 0 . 4 )} 0.0635$ & $(0.7,0.4)$ \\
\hline $\mathbf{T}_{\mathbf{2}}$ & $\mathbf{( 0 . 4 , 0 . 7 )} 0.056$ & $\mathbf{( 0 . 5 7 4 7 5 , 0 . 5 7 1 4 2 )} 0.07$ & $\mathbf{( 0 . 6 , 0 . 5 )} 0.026$ & 0.07 & $(0.8,0.2)$ \\
\hline $\mathbf{T}_{\mathbf{3}}$ & 0.074 & $\mathbf{( 0 . 4 8 8 2 8 , 0 . 4 5 2}) 0.0815$ & 0.06 & $\mathbf{( 0 . 8 6 6 3 2 , 0 . 1 9 0 4 7 )} 0.09$ & $(0.9,0.1)$ \\
\hline Requirement & $(0.4,0.7)$ & $(0.7,0.3)$ & $(0.6,0.5)$ & $(0.3,0.8)$ & \\
\hline
\end{tabular}

The initial fuzzy transportation cost $=(0.46133,0.75227)$ units.

Now to obtain the optimal solution of given Pythagorean Species fuzzy transportation problem, Modi method has applied in the IFBFS.

Using Modi method, the optimal basic variables and optimal solution is as follows

$$
\begin{aligned}
& b_{11}=(0.4,0.7), b_{12}=(0.62678,0.57142), b_{23}=(0.6,0.5), \\
& b_{24}=(0.66142,0.39994), b_{32}=(0.39997,0.52492), b_{34}=(0.87965,0.19048)
\end{aligned}
$$

Hence, the optimal solution using proposed method $=(0.45608,0.72097)$ units.

It is clear from the above calculation that if the species in $T_{1}$ transported to $J_{1}$ and $J_{2}$, species in $T_{2}$ transported to $\mathrm{J}_{3}$ and $\mathrm{J}_{4}$ and species in $\mathrm{T}_{3}$ transported to $\mathrm{J}_{2}$ and $\mathrm{J}_{4}$; the obtained cost of transportation is optimal.

The optimal transportation cost found out in fuzzy number, showing that there is no loss of any information. 
11. Comparative Study

\subsection{Comparison based on Allocation}

This section describes the comparison for allocation obtained by proposed schemes. Place of allocation is compared for example 1 and example 2 (Kumar et al., 2019; Umamageswari and Uthra, 2020) in Table 7 and Table 8.

Table 7. Table of comparison for allocation for example 1.

\begin{tabular}{|c|c|c|c|c|c|c|c|c|c|c|c|c|}
\hline & \multicolumn{3}{|c|}{$\mathrm{S}_{1}$} & \multicolumn{3}{|c|}{$S_{2}$} & \multicolumn{3}{|c|}{$\mathbf{S}_{3}$} & \multicolumn{3}{|c|}{$\mathbf{S}_{4}$} \\
\hline & $\begin{array}{l}\text { Kumar } \\
\text { et al., } \\
(2019)\end{array}$ & $\begin{array}{l}\text { Umamages } \\
\text { wari and } \\
\text { Uthra, } \\
(2020)\end{array}$ & Prop.approach & $\begin{array}{l}\text { Kumar } \\
\text { et al., } \\
(2019)\end{array}$ & $\begin{array}{l}\text { Umamageswari } \\
\text { and } \\
\text { (2020) Uthra, }\end{array}$ & $\begin{array}{l}\text { Prop. } \\
\text { Approach }\end{array}$ & $\begin{array}{l}\text { Kumar } \\
\text { et al., } \\
\text { (2019) }\end{array}$ & $\begin{array}{l}\text { Umamageswari } \\
\text { and } \\
\text { (2020) Uthra, }\end{array}$ & $\begin{array}{l}\text { Prop. } \\
\text { Approach }\end{array}$ & $\begin{array}{l}\text { Kumar } \\
\text { et al., } \\
\text { (2019) }\end{array}$ & $\begin{array}{l}\text { Umamageswari } \\
\text { and Uthra, } \\
\text { (2020) }\end{array}$ & $\begin{array}{l}\text { Prop. } \\
\text { Approach }\end{array}$ \\
\hline $\mathbf{R}_{1}$ & $x$ & $x$ & $\checkmark$ & $x$ & $\checkmark$ & $x$ & $\checkmark$ & $x$ & $x$ & $x$ & $\checkmark$ & $\checkmark$ \\
\hline $\mathbf{R}_{2}$ & $\checkmark$ & $x$ & $x$ & $x$ & $\checkmark$ & $\checkmark$ & $\checkmark$ & $\checkmark$ & $x$ & $\sqrt{2}$ & $x$ & $\sqrt{2}$ \\
\hline $\mathbf{R}_{3}$ & $x$ & $\checkmark$ & $x$ & $\checkmark$ & $x$ & $\checkmark$ & $x$ & $\sqrt{ }$ & $\sqrt{2}$ & $\sqrt{2}$ & $x$ & $x$ \\
\hline
\end{tabular}

In Table 7 , it can be seen that using the proposed method, $R_{1}$ maps $S_{1}$ and $S_{4}$ in which $S_{1}$ has minimum transportation cost in the first row, $R_{2}$ maps $S_{2}$ and $S_{4}$ in which $S_{2}$ has minimum transportation cost in the second row and $R_{3}$ maps $S_{2}$ and $S_{3}$ in which $S_{3}$ has minimum transportation cost in the third row. This shows that, most of the time, the proposed method allocates those cells which have minimum transportation cost for supplying one unit which results in overall minimizing the transportation cost. On the other hand, existing methods allocates those cells which have high transportation cost.

Table 8. Table of comparison for allocation for example 2.

\begin{tabular}{|c|c|c|c|c|c|c|c|c|c|c|c|c|}
\hline & \multicolumn{3}{|c|}{$\mathbf{J}_{\mathbf{1}}$} & \multicolumn{3}{|c|}{$\mathbf{J}_{\mathbf{2}}$} & \multicolumn{3}{|c|}{$\mathbf{J}_{3}$} & \multicolumn{3}{|c|}{$\mathbf{J}_{4}$} \\
\hline & $\begin{array}{l}\text { Kumar } \\
\text { et al., } \\
\text { (2019) }\end{array}$ & $\begin{array}{l}\text { Umamageswari } \\
\text { and Uthra, } \\
\text { (2020) }\end{array}$ & $\begin{array}{l}\text { Prop. } \\
\text { approach }\end{array}$ & $\begin{array}{l}\text { Kumar et } \\
\text { al., } \\
\text { (2019) }\end{array}$ & $\begin{array}{l}\text { Umamageswari } \\
\text { \& Uthra, (2020) }\end{array}$ & $\begin{array}{l}\text { Prop. } \\
\text { Approach }\end{array}$ & $\begin{array}{l}\text { Kumar } \\
\text { et al., } \\
\text { (2019) }\end{array}$ & $\begin{array}{l}\text { Umamageswari } \\
\text { and Uthra, } \\
\text { (2020) }\end{array}$ & $\begin{array}{l}\text { Prop. } \\
\text { Approach }\end{array}$ & $\begin{array}{l}\text { Kumar } \\
\text { et al., } \\
\text { (2019) }\end{array}$ & $\begin{array}{l}\text { Umamageswari } \\
\text { and Uthra, } \\
(2020)\end{array}$ & $\begin{array}{l}\text { Prop. } \\
\text { Approach }\end{array}$ \\
\hline$T_{1}$ & $V$ & $\checkmark$ & $\checkmark$ & $x$ & $x$ & $V$ & $x$ & $x$ & $x$ & $x$ & $x$ & $x$ \\
\hline $\mathbf{T}_{2}$ & $x$ & $x$ & $x$ & $x$ & $x$ & $x$ & $v$ & $\checkmark$ & $v$ & $x$ & $v$ & $v$ \\
\hline$T_{3}$ & $x$ & $x$ & $x$ & $v$ & $v$ & $v$ & $v$ & $x$ & $x$ & $x$ & $v$ & $v$ \\
\hline
\end{tabular}

In Table 8 , it can be seen that using the proposed method, $T_{1}$ maps to $J_{1}$ and $J_{2}$ in which $J_{1}$ has minimum transportation cost in the first row, $T_{2}$ maps to $J_{3}$ and $J_{4}$ in which $J_{3}$ has minimum transportation cost in the second row and $T_{3}$ maps to $J_{2}$ and $J_{4}$. This shows that, most of the time, the proposed method allocates those cells which have minimum transportation cost for supplying one unit which results in overall minimizing the transportation cost. On the other hand, existing methods allocates those cells which have high transportation cost.

\subsection{Comparison based on Optimal Transportation Cost}

Optimal transportation cost is compared for example 1 and example 2 (Kumar et al., 2019; Umamageswari and Uthra, 2020) shown in Table 9 and Table 10.

Table 9. Comparison based on optimal transportation cost for example 1.

\begin{tabular}{|l|l|l|l|l|}
\hline Method & Ranking Technique Used & Information Lose & Solution in fuzzy & Cost of transportation \\
\hline Kumar et al., (2019) & Yes & Yes & No & 11.45 \\
\hline $\begin{array}{l}\text { Umamageswari and } \\
\text { Uthra, (2020) }\end{array}$ & Yes & Yes & No & 24.25 \\
\hline Proposed Scheme & No & No & Yes & $(0.99999,0)$ \\
\hline
\end{tabular}


Table 10. Comparison based on optimal transportation cost for example 2 .

\begin{tabular}{|l|l|l|l|l|}
\hline Method & Ranking Technique Used & Information Lose & Solution in fuzzy & Cost of transportation \\
\hline Kumar et al., (2019) & Yes & Yes & No & 0.016955 \\
\hline $\begin{array}{l}\text { Umamageswari and } \\
\text { Uthra, (2020) }\end{array}$ & Yes & Yes & No & 0.056352 \\
\hline Proposed Scheme & No & No & Yes & $(0.45608,0.72097)$ \\
\hline
\end{tabular}

\section{Conclusions and Future Directions}

The relocation of a variety of special and rare species in optimized time and cost is always being a concern for mankind. The uncertainty in data makes it more challenging to handle. In the present study, a special class of fuzzy transportation problem named as Pythagorean fuzzy species transportation problem (PFSTP) is optimized. In this work, two novel schemes have suggested to transport species from one domain to another domain. PFNs are used as parametric values. In order to evaluate the IFBFS of the PFSTP, the first proposed scheme is used. Secondly, for finding optimal transportation cost, another proposed scheme is applied. In literature, there is no technique for finding the optimal solution of Pythagorean FTP in fuzzy form. However, proposed method provides the optimal solution in fuzzy form and obtaining optimal transportation cost in fuzzy values reduces the possibility of losing any type of information. To check the effectiveness of the proposed algorithms, comparison has done with the existing methods. The present scheme is easy to apply in real-life situations.

The proposed study is limited to type-1 and type-2 Pythagorean species fuzzy transportation problem and in future this study can be extended to solve fully fuzzy species Pythagorean transportation problems. Additionally, a variety of other transportation problems like Neutrosophic fuzzy transportation problem in fuzzy environment can be optimized using proposed technique.

\section{Conflict of Interest}

The authors declare that there is no conflict of interest for this publication.

\section{Acknowledgments}

The authors extend their appreciation to the anonymous reviewers for their valuable suggestions.

\section{References}

Akilbasha, A., Pandian, P., \& Natarajan, G. (2018). An innovative exact method for solving fully interval integer transportation problems. Informatics in Medicine Unlocked, 11, 95-99.

Arora, J. (2018). An algorithm for interval-valued fuzzy fractional transportation problem. Skit Research Journal, 8(1), 71-75.

Atanassov, K.T. (1986). Intuitionistic fuzzy sets. Fuzzy Sets Systems, 20(1), 87-96.

Bellman, R.E., \& Zadeh, L.A. (1970). Decision-making in a fuzzy environment. Management Science, 17(4), 141-170. https://doi.org/10.1287/mnsc.17.4.B141.

Bharati, S.K., \& Singh, S.R. (2018). Transportation problem under interval-valued intuitionistic fuzzy environment. International Journal of Fuzzy Systems, 20(5), 1511-1522.

Bisht, D.C.S., \& Srivastava, P.K. (2019a). Fuzzy optimization and decision making. In: Ram, M., Davim, J.P. (eds) Advanced Fuzzy Logic Approaches in Engineering Science. IGI Global, Pennsylvania, USA, pp. 310-326. 
Bisht, D.C.S., \& Srivastava, P.K. (2019b). One-point conventional model to optimize trapezoidal fuzzy transportation problem. International Journal of Mathematical, Engineering and Management Sciences, 4(5), 1251-1263.

Bisht, D.C.S., \& Srivastava, P.K. (2018). Trisectional fuzzy trapezoidal approach to optimize interval data based transportation problem. Journal of King Saud University-Science, 32(1), 195-199.

Bisht, D.C.S., Srivastava, P.K., \& Ram, M. (2018). Role of fuzzy logic in flexible manufacturing system. In: Ram, M., Davim, J.P. (eds) Diagnostic Techniques in Industrial Engineering. Springer, Cham, pp. 233243.

Bisht, D., \& Srivastava, P.K. (2017). A unique conversion approach clubbed with a new ranking technique to optimize fuzzy transportation cost. In AIP Conference Proceedings (Vol. 1897, No. 1, p. 020023). AIP Publishing LLC. Uttar Pradesh, India.

Chanas, S., Kolodziejczyk, W., \& Machaj, A. (1984). A fuzzy approach to the transportation problem. Fuzzy Sets and Systems, 13(3), 211-221.

Chanas, S., \& Kuchta, D. (1996). A concept of the optimal solution of the transportation problem with fuzzy cost coefficients. Fuzzy Sets and Systems, 82(3), 299-305.

Chhibber, D., Bisht, D.C.S., \& Srivastava, P.K. (2019). Ranking approach based on incenter in triangle of centroids to solve type-1 and type-2 fuzzy transportation problem. In AIP Conference Proceedings (Vol. 2061, No. 1, p. 020022). AIP Publishing LLC. Noida, India.

Dinagar, D.S., \& Keerthivasan, R. (2018). Solving fuzzy transportation problem using modified best candidate method. Journal of Computer and Mathematical Sciences, 9(9), 1179-1186.

Dinagar, D.S., \& Palanivel, K. (2009). The transportation problem in fuzzy environment. International Journal of Algorithms, Computing and Mathematics, 2(3), 65-71.

Ebrahimnejad, A. (2014). A simplified new approach for solving fuzzy transportation problems with generalized trapezoidal fuzzy numbers. Applied Soft Computing, 19, 171-176.

Ebrahimnejad, A. (2015). An improved approach for solving fuzzy transportation problem with triangular fuzzy numbers. Journal of Intelligent \& Fuzzy Systems, 29(2), 963-974.

Gani, A.N., \& Razak, K.A. (2006). Two stage fuzzy transportation problem. Journal of Physical Science, 10, 63-69.

Hashmi N., Jalil, S.A., \& Javaid, S. (2019). A model for two-stage fixed charge transportation problem with multiple objectives and fuzzy linguistic preferences. Soft Computing, 23, 12401-12415. https://doi.org/10.1007/s00500-019-03782-1

Hitchcock, F.L. (1941). The distribution of a product from several sources to numerous localities. Journal of Mathematics and Physics, 20(1-4), 224-230.

Kaur, A., \& Kumar, A. (2011). A new method for solving fuzzy transportation problems using ranking function. Applied Mathematical Modelling, 35(12), 5652-5661.

Kaur, A., \& Kumar, A. (2012). A new approach for solving fuzzy transportation problems using generalized trapezoidal fuzzy numbers. Applied Soft Computing, 12(3), 1201-1213.

Kumar, A., \& Kaur, A. (2011). Application of classical transportation methods for solving fuzzy transportation problems. Journal of Transportation Systems Engineering and Information Technology, 11(5), 68-80.

Kumar, R., Edalatpanah, S.A., Jha, S., \& Singh, R. (2019). A pythagorean fuzzy approach to the transportation problem. Complex and Intelligent Systems, 5(2), 255-263. 
Kundu, P., Kar, S., \& Maiti, M. (2014). Fixed charge transportation problem with type-2 fuzzy variables. Information Sciences, 255, 170-186.

Li, L., \& Lai, K.K. (2000). A fuzzy approach to the multiobjective transportation problem. Computers and Operation Research, 27(1), 43-57.

Liu, P., Yang, L., Wang, L., \& Li, S. (2014). A solid transportation problem with type-2 fuzzy variables. Applied Soft Computing, 24, 543-558.

Liu, S.T., \& Kao, C. (2004). Solving fuzzy transportation problems based on extension principle. European Journal of Operational Research, 153(3), 661-674.

Mathur, N., \& Srivastava, P.K. (2019). A pioneer optimization approach for hexagonal fuzzy transportation problem. In AIP Conference Proceedings (Vol. 2061, No. 1, p. 020030). AIP Publishing LLC. Noida, India.

Mathur, N., Srivastava, P.K., \& Paul, A. (2016). Trapezoidal fuzzy model to optimize transportation problem. International Journal of Modeling, Simulation, and Scientific Computing, 7(3), 1650028.

Mathur, N., Srivastava, P.K., \& Paul, A. (2018). Algorithms for solving fuzzy transportation problem. International Journal of Mathematics in Operational Research, 12(2), 190-219.

Madden, M.E., Runge, M.C., Possingham, H.P., \& Martin, T.G. (2011). Optimal timing for managed relocation of species faced with climate change. Nature Climate Change, 1(5), 261-265.

Nagar, P., Srivastava, A., \& Srivastava, P.K. (2019). Optimization of species transportation via an exclusive fuzzy trapezoidal centroid approach. Mathematics in Engineering, Science and Aerospace, 10(2), 271280.

Ngastiti, P.T.B., Surarso, B., \& Sutimin, S. (2018). Zero point and zero suffix methods with robust ranking for solving fully fuzzy transportation problems. Journal of Physics Conference Series, 1022, 01-09.

Olden, J.D., Kennard, M.J., Lawler, J.J., \& Poff, N.L. (2011). Challenges and opportunities in implementing managed relocation for conservation of freshwater species. Conservation Biology, 25(1), 40-47.

Pandian, P., \& Natarajan, G. (2010). A new method for finding an optimal solution of fully interval integer transportation problems. Applied Mathematical Sciences, 4(37), 1819-1830.

Samuel, A.E., \& Venkatachalapathy, M. (2013). A simple heuristic for solving generalized fuzzy transportation problems. International Journal of Pure and Applied Mathematics, 83(1), 91-100.

Sheean, V.A., Manning, A.D., \& Lindenmayer, D.B. (2012). An assessment of scientific approaches towards species relocations in Australia. Austral Ecology, 37(2), 204-215.

Singh, S.K., \& Yadav, S.P. (2016). A new approach for solving intuitionistic fuzzy transportation problem of type-2. Annals of Operation Research, 243, 349-363.

Srivastava, P.K., \& Bisht, D.C.S. (2018). Dichotomized incenter fuzzy triangular ranking approach to optimize interval data-based transportation problem. Cybernetics and Information Technologies, 18(4), 111-119.

Srivastava, P.K., \& Bisht, D.C.S. (2019). Recent trends and applications of fuzzy logic. In: Ram, M., Davim, J.P. (eds) Advanced Fuzzy Logic Approaches in Engineering Science. IGI Global, Pennsylvania, USA, pp. 327-340.

Umamageswari, R.M., \& Uthra, G. (2020). A pythagorean fuzzy approach to solve transportation problem. Adalya Journal, 9(1), 1301-1308.

Yager, R.R. (2014). Pythagorean membership grades in multicriteria decision making. IEEE Transactions on Fuzzy Systems, 22(4), 958-965. 
Yager, R.R., \& Abbasov, A.M. (2013). Pythagorean membership grades, complex numbers, and decision making. International Journal of Intelligent Systems, 28(5), 436-452.

Yager, R.R. (2013). Pythagorean fuzzy subsets. In 2013 Joint IFSA World Congress and NAFIPS Annual Meeting (pp. 57-61). IEEE. Edmonton, Canada.

Zadeh, L.A. (1965). Fuzzy sets. Information and Control, 8(3), 338-353.

Zhang, X. (2016). A novel approach based on similarity measure for pythagorean fuzzy multiple criteria group decision making. International Journal of Intelligent Systems, 31(6), 593-611.

Zimmermann, H.J. (1978). Fuzzy programming and linear programming with several objective functions. Fuzzy Sets and Systems, 1(1), 45-55. 